\title{
COMMUNITY OUTREACH THROUGH GENOMICS EDUCATION PARTNERSHIP
}

$\underline{\text { Ramana Madupu }}{ }^{1}$, Anne G. Rosenwald ${ }^{2}$, A. Malcolm Campbell ${ }^{3}$, Satish C Bhalla ${ }^{4}$, Granger Sutton $^{1}$

1.The J. Craig Venter Institute, Rockville, MD 20850 2.Georgetown University, Washington DC 200573.Davidson College, Davidson, NC 28036 4.Johnson C Smith University, Charlotte, NC 28216

Abstract:JCVI has recently partnered with undergraduate university faculty to expand the scope of education and outreach program as part of the NIAID's BRC initiative, by joining forces with faculty members participating in the Genomics Education Partnership (GEP). The goal of the GEP is to provide opportunities for undergraduate students to participate in genomics research and gain hands on experience. Faculty members trained on annotation methodologies and tools during the Prokaryotic Annotation Workshop conducted at JCVI, impart their knowledge in the classroom as part of the semester course. As a pilot project, we are currently collaborating with 3 groups lead by a faculty member, spread across 3 universities in the community curation of bacterial genomes. Each participating undergraduate group collectively annotates a specific bacterial genome that was sequenced at JCVI and run through the automatic annotation pipeline. Remote access to genome sequence data, pre-computed gene predictions, search results, automatic annotation and bioinformatics analysis is provided through our web-based manual annotation tool, MANATEE. The students log into JCVI genome databases with user specific ids and password and learn to annotate single genes, entire metabolic pathways leading to analysis of a question that may be unique to the genome being analyzed. Users of the genome data receive dedicated support and guidance from our in house annotation experts on the usage of JCVI's tools and annotation methodologies. Through this exercise, the undergraduate students are introduced to concepts of genomics and

bioinformatics and gain deeper understanding of the concepts of cellular metabolism and disease pathology, which may lead them to making scientific research their career path. Some groups are focusing on genome specific pathways and plan to conduct wet lab experiments to understand unique genome features. We are highly encouraged that this model of web based, remote access, community annotation has been successful and propose to leverage the community of annotators to update annotations of pathogen genomes in Pathema-BRC.

$\checkmark$ Each undergrad group is provided remote access to a bacterial genome sequence and data

$\checkmark$ Each student is assigned a specific functional role category or metabolic pathway to annotate

$\checkmark$ Students login to JCVI's database with specific user ids

Access to genome data through Manatee

Manatee is a web-based manual annotation tool for accessing and editing

annotation data

Manatee draws information from an underlying database for its displays
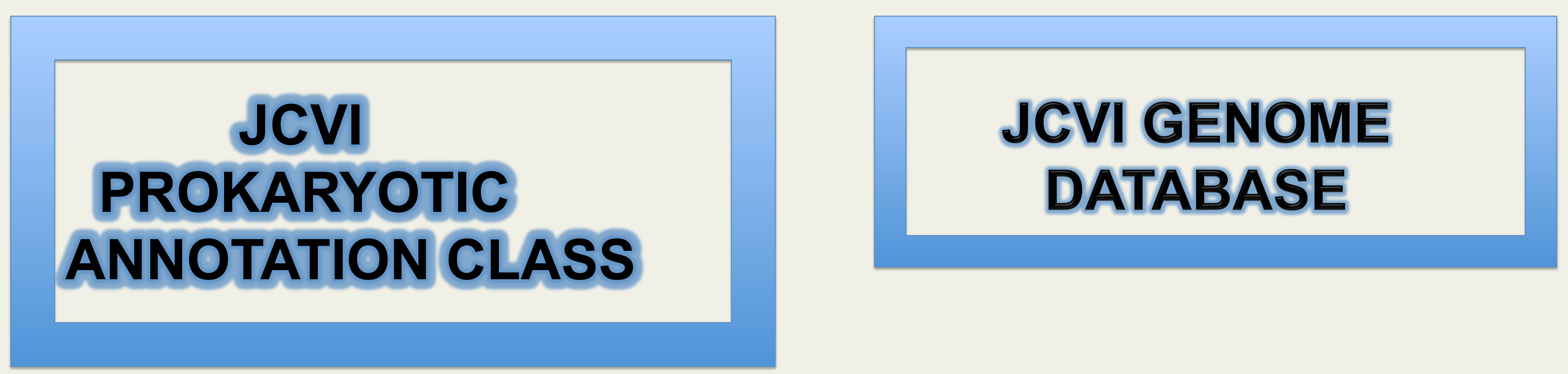

\section{University Faculty \\ Trained in Annotation Methodologies and Tools}

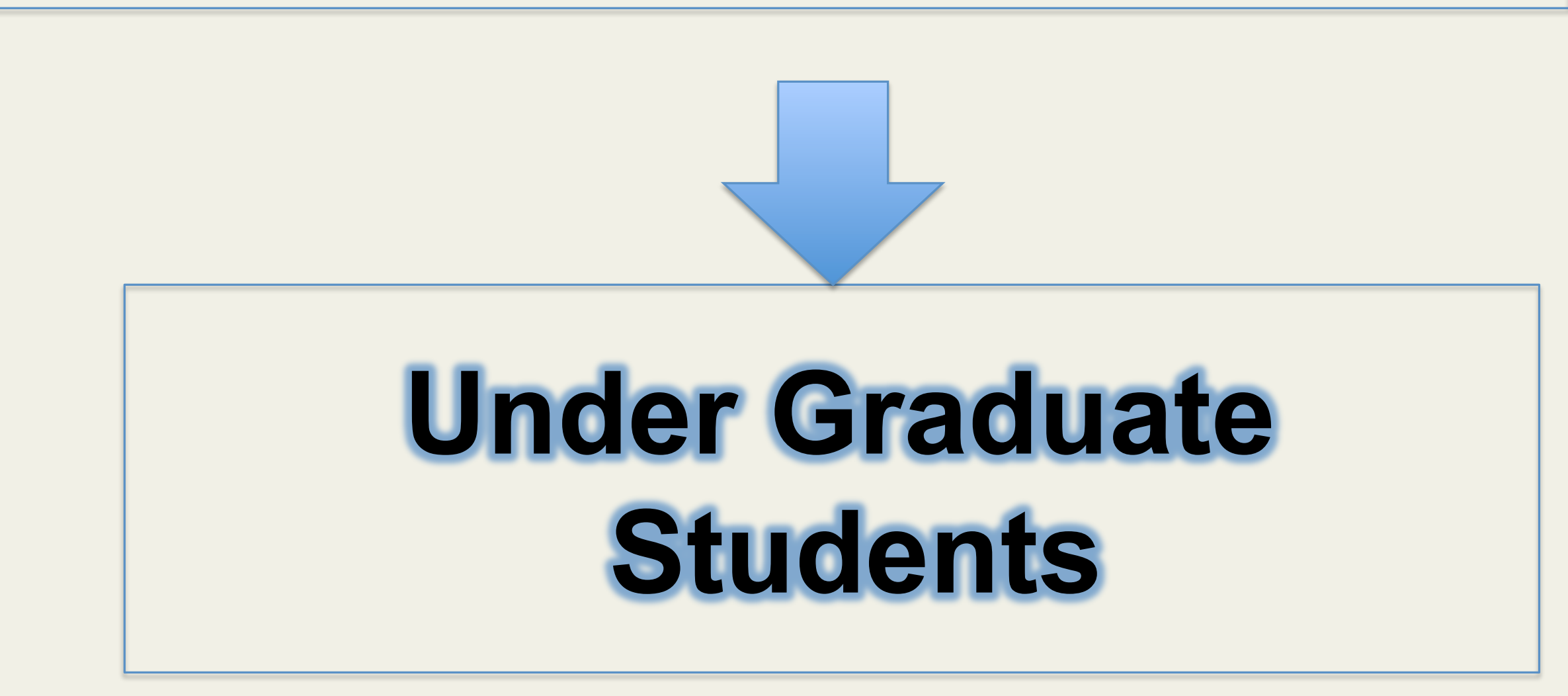

MANATEE

Manatee sends information entered by annotators to the underlying database for storage

Manatee depends on JCVI's database structure

Multiple users can access the same database from different computers

when Manatee is run on a server

Student work is rated and Annotations reviewed

$\checkmark$ Conduct simple lab experiments to understand Genome Physiology

Acknowledgments: This project has been funded in whole or in part with Federal funds from the National Institute of Allergy and Infectious Diseases, National Institutes of Health, Department of Health and Human Services, under Contract No. HHSN266200400038C 